\title{
Identification of a novel splice mutation in CTNNB1 gene in a Chinese family with both severe intellectual disability and serious visual defects
}

\author{
Hui Wang ${ }^{1} \cdot$ Yiqi Zhao ${ }^{1} \cdot$ Liwei Yang ${ }^{1}$ (D) Shuai Han $^{2} \cdot$ Ming $\mathbf{Q i}^{2,3,4}$ \\ Received: 28 September 2018 / Accepted: 5 March 2019 / Published online: 30 March 2019 \\ (C) The Author(s) 2019
}

\begin{abstract}
The $C T N N B 1$ gene encode the $\beta$-catenin protein which is a core unit of the cadherin/catenin multiprotein complex. The loss-offunction mutation of the $C T N N B 1$ gene recently has been confirmed as a cause of intellectual disability. Previous studies have found that patients with $C T N N B 1$ gene mutation may have other clinical manifestation such as microcephaly, abnormal facial features, motor and language delays, and mild visual defects. Here, we reported a 27 -year-old Chinese pregnant woman with a severe intellectual disability and serious visual defects who was detected with a novel splice mutation (c.734+1G $>\mathrm{A})$ in $C T N N B 1$ gene by whole-exome sequencing and confirmed by Sanger sequencing. Further investigation showed that the variant was inherited from her mother with similar phenotypes. This report not only helps to expand the mutant spectrum of the CTNNBI gene but also prompts a new insight into genetic diagnosis in patients with both serious intellectual disability and visual defects.
\end{abstract}

Keywords $C T N N B 1$ gene $\cdot$ Intellectual disability $\cdot$ Visual defects $\cdot$ Splice mutation

\section{Introduction}

The $C T N N B 1$ gene encodes the $\beta$-catenin protein which is a sub-unit of the cadherin/catenin multiprotein complex. Previous studies find $C T N N B 1$ mutation related to several

Wang Hui and Zhao Yiqi contributed equally to this work.

Electronic supplementary material The online version of this article (https://doi.org/10.1007/s10072-019-03823-5) contains supplementary material, which is available to authorized users.

Liwei Yang

yanglw1967@163.com

1 Department of Obstetrics, Zhejiang Provincial People's Hospital, People's Hospital of Hangzhou Medical College, No. 158, Shangtang Road, Hangzhou, Zhejiang Province, China

2 Department of Cell Biology and Medical Genetics, School of Medicine, Zhejiang University, Hangzhou, China

3 Assisted Reproduction Unit, Department of Obstetrics and Gynecology, Sir Run Run Shaw Hospital, School of Medicine, Key Laboratory of Reproductive Dysfunction Management of Zhejiang Province, Zhejiang University, Hangzhou, China

4 Department of Pathology and Laboratory Medicine, University of Rochester Medical Center, Rochester, NY, USA cancers [1]. Recently, a large-scale sequencing firstly identified $C T N N B 1$ loss-of-function mutations as the cause of intellectual disability (ID) [2]. Other studies report different mutations in patients which phenotypes include ID, craniofacial anomalies, speech delay microcephaly, and mild visual disturbances like strabismus and hyperopia [3-5]. Our study describes a novel mutation of the CTNNB1 gene in a family who presented with serious ID and visual disturbances.

\section{Case report}

The proband was a 27-years-old Chinese pregnant woman who attended to our department at 20th gestational week for prenatal diagnosis because of the possible maternal ID. She also had severe esotropia, involuntary rotation of the head, and significant motor delay and language retardation (Fig. 1a), which appeared and got worsen since her birth. Neurologist and ophthalmologist were invited for a professional examination.

The neurologic examination found she had peripheral hypertonia with deep tendon hyperreflexia and Babinski sign positive, ataxic gait, and paroxysmal dystonic of head and neck, especially right-side rotation of head (Online Resource 


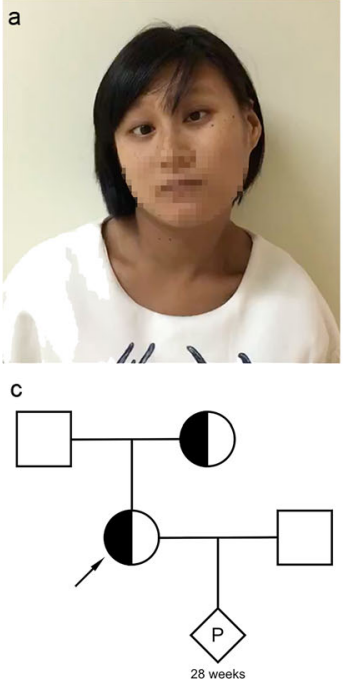

Proband
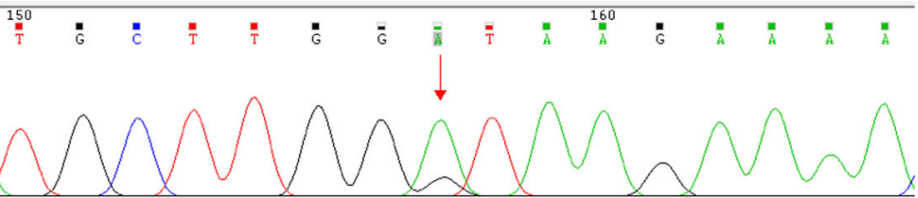

Mother

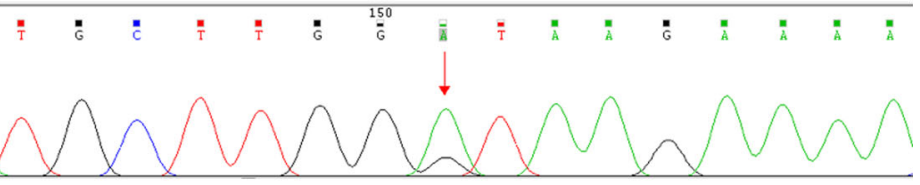

Wild type

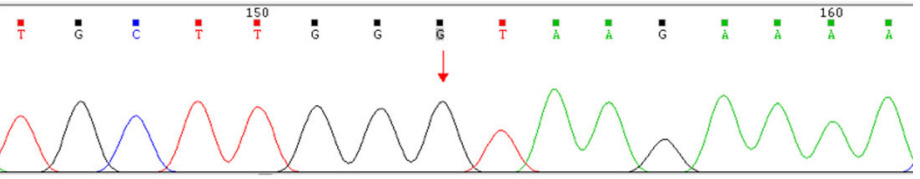

Fig. 1 Photographs of the proband, mutations in the CTNNB1 gene, and the pedigrees of the families. a The proband had mildly abnormal craniofacial features with face involuntary movements, severe esotropia, rotation of the head, and significant motor delay and language retardation.

1). Ceruloplasmin test, immunologic tests, brain MRI, and routine laboratory tests revealed nothing remarkable. Electroencephalograph (EEG) was mildly abnormal without typical epileptiform discharge and electromyography (EMG) was normal. Wechsler intelligence test showed severe ID with IQ score 28. The ophthalmologist found the woman had serious esotropia of both eyes, no reaction to light, and a complete lack of vision accompanied with nystagmus, lens, and vitreous opacities on oculus sinister. Complete retinal detachment and eyeball atrophy on her left eye were confirmed by fundus ultrasound.

For further investigation, whole-genome low-coverage sequencing (WGLCS) and whole-exome sequencing (WES) were performed to detect the potential pathogenic factors besides Karyotype (Online Resource 2). A novel mutation (c. $734+1 \mathrm{G}>\mathrm{A})$ in the $C T N N B 1$ gene was detected by WES and confirmed by Sanger sequencing (Fig. 1b).

To our interest, we learned that the proband's mother has similar but slighter clinical manifestations (Fig. 1c; Online Resource 3). She was invited to our hospital for detailed examinations. She was 49 years old with ID (IQ score 40) and had similar neurological clinical manifestations (Fig. 2a, b) and unremarkable brain MRI and EEG. Ophthalmologic examination indicated impaired visual acuity (logarithmic visual acuity chart 0.08 in OD and 0.1 in OS) and esotropia. Fundus fluorescein angiography was then performed and displayed the exudative vitreoretinopathy (EVR) on the temporal side in both of her eyes (Fig. 2c). Sanger sequencing was carried out and confirmed the same splice mutation $(\mathrm{c} .734+1 \mathrm{G}>\mathrm{A})$ in the CTNNB1 gene in the proband's mother (Fig. 1c).

The splice mutation (c.734+1G $>$ A) on CTNNB 1 gene has not been reported by any research or recorded by any related b Sanger sequence showed a heterozygous splice mutation (c.734+ $1 \mathrm{G}>\mathrm{A}$ ) in the proband and her mother. Red arrows, mutant bases. $\mathbf{c}$ The pedigrees of the families

gene mutation database, such as HGMD, Clinvar, and LOVD and leads to a truncated CTNNB1 protein of 245 in comparison with the wide-type sequence of 781 amino acids. We performed three-dimensional modeling change of the truncated CTNNB1 protein (Fig. 2d), which loses the very important functional domain interactive with BCL9 (B cell CLL/ lymphoma 9) and SCRIB indicated by Unipro database. As BCL9 and SCRIB both participate in the Wnt signaling pathway, so we infer that the splice mutation $(\mathrm{c} .734+1 \mathrm{G}>\mathrm{A})$ affect the structure and function of a $\beta$-catenin protein which may influence the Wnt signaling pathway and causing a series of clinical manifestations. So, our further research should elucidate how the mutation affects the function of $\beta$-catenin and the precise pathogenic molecular mechanisms.

\section{Discussion}

The CTNNB1 gene encodes a $\beta$-catenin protein which is a central component of the cadherin/catenin complex. $\beta$ catenin protein participates in cell adhesion and nuclear signaling which mainly involves in the Wnt protein-mediated signal pathway. Previous studies declare CTNNB1 mutation is related to several cancers such as hepatocellular carcinoma, medulloblastoma, ovarian cancer, and pilomatricoma [1]. It was firstly connected to ID since 2012 by De Ligt J [2] and then, a series of studies report more than 20 loss-functionmutations of the CTNNB1 gene which may lead to ID [6]. As known, human $\beta$-catenin contains 781 amino acids and is subdivided into three domains: an amino-terminal domain (NTD), a central region containing 12 armadillo (ARM) repeats (residues 138-664), and a carboxy-terminal domain 


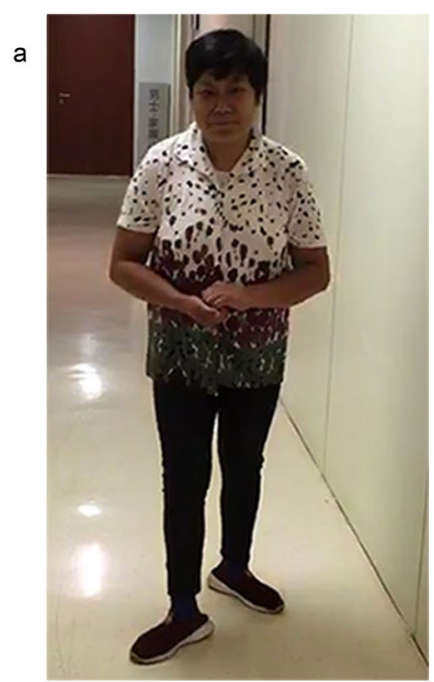

b

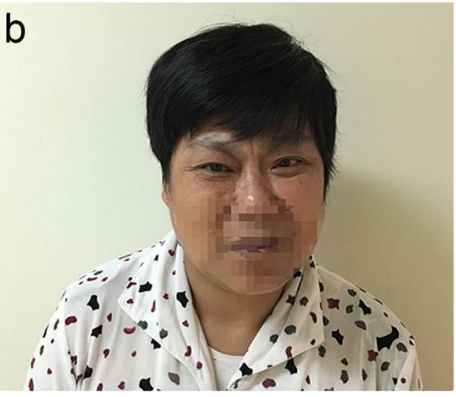

Fig. 2 Photographs of proband's mother and her fundus fluorescein angiography, expected effects on amino acid sequences, and protein functionality. a and $\mathbf{b}$ The proband's mother had similar but slighter abnormal craniofacial features like a grimace, esotropia, rotation of the head, and ataxic gait. c Fundus fluorescein angiography showed exudative vitreoretinopathy on the temporal side in both eyes of

(CTD). However, by which mechanism, the truncated protein identified by this study may influence the Wnt signaling; it is worth further research.

Phenotypic characteristics of CTNNB1 mutation patients include ID, postnatal microcephaly, mild craniofacial particularities, and neurological disorders such as peripheral hypertonia, motor, and language development delay (Online Mendelian Inheritance in Man, OMIM\# 615075). Mild visual disturbances were also reported such as astigmatism, hyperopia, and strabismus. Our case also indicated similar features but the proband suffers from complete retinal detachment and her mother also has severely impaired visual acuity. Panagiotou et al. found the CTNNB1 gene can result in exudative vitreoretinopathy with no neurological disorders [7]. However, one 3-year-old Chinese boy initially presented with EVR showed facial dysmorphism and global developmental delay during follow-up and WES identified a de novo 1-bp insertion (c.1434_1435insC, Glu479ArgfsTer18) in CTNNB1 gene (OMIM *116806.0023). Niu Li et al. also reported a 15-month-old Chinese boy with retinal detachment and development delay, who was negative for mutation related to the exudative vitreoretinopathy but a heterozygous nonsense mutation (c.1627C>T, p. Gln558X) in CTNNB1 gene was found [6]. Combined with our case, we suppose that CTNNB1 gene mutation not only can cause ID or EVR singly but also may synchronously result in these series phenotypes.

In conclusion, our study identifies a novel heterozygous splice mutation in the $C T N N B 1$ gene $($ c. $734+1 \mathrm{G}>\mathrm{A})$ in a Chinese family with both severe ID and visual disturbances. It helps to expand the mutant spectrum of the CTNNB1 gene and confirms that different types of mutation in CTNNB1 may be associated with ID and visual disturbances.

Acknowledgments The authors would like to thank Guo Shunyuan, CHI Xin Chang, and Qiu Yaju for their comprehensive physical examination and critically reviewing of the manuscript. We are also grateful for all individuals who participated in this study.

\section{Compliance with ethical standards}

Conflict of interest The authors declare that they have no conflict of interest. 
Ethical approval All procedures performed in studies involving human participants were in accordance with the ethical standards of the institutional and national research committee and with the 1964 Helsinki Declaration and its later amendments or comparable ethical standards.

Informed consent Written informed consent was obtained from all individual participants included in the study for photograph and publication.

Open Access This article is distributed under the terms of the Creative Commons Attribution 4.0 International License (http:// creativecommons.org/licenses/by/4.0/), which permits unrestricted use, distribution, and reproduction in any medium, provided you give appropriate credit to the original author(s) and the source, provide a link to the Creative Commons license, and indicate if changes were made.

\section{References}

1. Anastas JN, Moon RT (2013) WNT signalling pathways as therapeutic targets in cancer. Nat Rev Cancer 13:11-26. https://doi.org/10. 1038/nrc3419

2. De Ligt J, Willemsen MH, van Bon BW, Kleefstra T, Yntema HG, Kroes T, Vulto-van Silfhout AT, Koolen DA, de Vries P, Gilissen C, del Rosario M, Hoischen A, Scheffer H, de Vries BB, Brunner HG, Veltman JA, Vissers LE (2012) Diagnostic exome sequencing in persons with severe intellectual disability. N Engl J Med 367: 1921-1929. https://doi.org/10.1056/NEJMoa1206524

3. Tucci V, Kleefstra T, Hardy A, Heise I, Maggi S, Willemsen MH, Hilton H, Esapa C, Simon M, Buenavista MT, McGuffin LJ, Vizor L, Dodero L, Tsaftaris S, Romero R, Nillesen WN, Vissers LE, Kempers MJ, Vulto-van Silfhout AT, Iqbal Z, Orlando M,
Maccione A, Lassi G, Farisello P, Contestabile A, Tinarelli F, Nieus T, Raimondi A, Greco B, Cantatore D, Gasparini L, Berdondini L, Bifone A, Gozzi A, Wells S, Nolan PM (2014) Dominant $\beta$-catenin mutations cause intellectual disability with recognizable syndromic features. J Clin Invest 124:1468-1482. https:// doi.org/10.1172/JCI70372

4. Kuechler A, Willemsen MH, Albrecht B, Bacino CA, Bartholomew DW, van Bokhoven H, van den Boogaard MJ, Bramswig N, Büttner C, Cremer K, Czeschik JC, Engels H, van Gassen K, Graf E, van Haelst M, He W, Hogue JS, Kempers M, Koolen D, Monroe G, de Munnik S, Pastore M, Reis A, Reuter MS, Tegay DH, Veltman J, Visser G, van Hasselt P, Smeets EE, Vissers L, Wieland T, Wissink W, Yntema H, Zink AM, Strom TM, Lüdecke HJ, Kleefstra T, Wieczorek D (2015) De novo mutations in beta-catenin (CTNNB1) appear to be a frequent cause of intellectual disability: expanding the mutational and clinical spectrum. Hum Genet 134:97-109. https:// doi.org/10.1007/s00439-014-1498-1

5. Dubruc E, Putoux A, Labalme A, Rougeot C, Sanlaville D, Edery P (2014) A new intellectual disability syndrome caused by CTNNB 1haploinsufficiency. Am J Med Genet 164A:1571-1575. https://doi.org/10.1002/ajmg.a.36484

6. Li N, Xu Y, Li G, Yu T, Yao R-e, Wang X, Wang J (2017) Exome sequencing identifies a de novo mutation of CTNNB1 gene in a patient mainly presented with retinal detachment, lens and vitreous opacities, microcephaly, and developmental delay: case report and literature review. Medicine 96:e6914. https://doi.org/10.1097/MD. 0000000000006914

7. Panagiotou ES, Sanjurjo Soriano C, Poulter JA, Lord EC, Dzulova D, Kondo H, Hiyoshi A, Chung BHY, Chu YWY, Lai CHY, Tafoya ME, Karjosukarso D, Collin RWJ, Topping J, Downey LM, Ali M, Inglehearn CF, Toomes C (2017) Defects in the cell signaling mediator beta-catenin cause the retinal vascular condition FEVR. Am J Hum Genet 100:960-968. https://doi.org/10.1016/j.ajhg.2017.05. 001

Publisher's note Springer Nature remains neutral with regard to jurisdictional claims in published maps and institutional affiliations. 